\title{
SOLVABLE SUBGROUPS IN PRIME RINGS
}

\author{
CHARLES LANSKI
}

\begin{abstract}
Let $R$ be a prime ring with center $Z$ and group of units $U$. The main theorem shows that any solvable normal subgroups of $U$ must lie in $Z$, provided that $R$ is not a domain, $Z$ is large enough, and that the $Z$-subalgebra generated by $U$ contains a nonzero ideal of $R$. One consequence is the determination of the structure of $R$ when $R$ has an involution and the subgroup of $U$ generated by the symmetric units is solvable.
\end{abstract}

Most results on rings whose solvable normal subgroups must be central have been restricted to division rings or to rings with idempotents (e.g. [3] and [4]). The purpose of this paper is to extend [4, Theorem 8.5, p. 569] on the centrality of such groups to the case of prime rings with zero divisors, and to use this extension to answer the question raised in [5] about the structure of those rings with involution for which the subgroup generated by all symmetric units is solvable. Examples presented in [5] show that domains can contain noncentral solvable normal subgroups, even if the domain is generated by its units; and that the same kind of subgroups can exist in prime rings with zero divisors, but not generated by their units. Since questions about units in a ring $R$ are really questions about units in the subring generated by the units of $R$, it is desirable to ensure that this subring is sufficiently large. To do this, we shall assume that the subring generated by the units of $R$ contains a nonzero ideal of $R$. Note that a prime ring $R$ will satisfy this assumption if its Jacobson radical is not zero or if $R$ contains a nontrivial idempotent (see [1, Lemma 1.10, p. 18]).

Throughout the paper, $R$ will denote a prime ring with 1 . Let $Z=Z(R)$ be the center of $R, U$ the group of units of $R$, and $\bar{U}$ the $Z$-subalgebra of $R$ generated by $U$. A nonempty subset $W \subset R$ is called invariant if $g^{-1} w g \in W$ for each $w \in W$ and $g \in U$. Our first result is trivial, but we shall use it a number of times.

LEMma. Let $W$ be an invariant subset of $R$ and let $x \in R$ so that either $W x=0$ or $x W=0$. If $\bar{U}$ contains a nonzero ideal of $R$, then either $W=0$ or $x=0$.

Proof. Assume $W x=0$. The invariance of $W$ implies that $g^{-1} W g \subset W$ for $g \in U$, and so $W \bar{U} x=0$. The assumption about $\bar{U}$ and the primeness of $R$ give the result. The case $x W=0$ is proved similarly.

Suppose that $N$ is a solvable normal subgroup of $U$ with derived series $1=$ $N^{(k+1)} \triangleleft N^{(k)} \triangleleft \cdots \triangleleft N$. Our method of proving that $N \subset Z$ will be to show that $N^{(k)} \subset Z$, and then show that $N^{(k-1)}$ is abelian. The first step is accomplished

Received by the editors September 22, 1980.

1980 Mathematics Subject Classification. Primary 16A25; Secondary 16A12, 16A28, 16 A48.

() 1981 American Mathematical Society 0002-9939/81/0000-0355/\$02.25 
by our first theorem. We shall use the notation $[x, y]=x y-y x$, and the identity $[x y, w]=x[y, w]+[x, w] y$, freely, without further reference.

THEOREM 1. Let $Z \neq \mathrm{GF}(2)$ and let $\bar{U}$ contain a nonzero ideal of $R$. If $W$ is $a$ commutative subring of $R$ which is invariant, then either $W \subset Z$ or $R$ is a domain.

Proof. Assume that $R$ is not a domain. Since $R$ is a prime ring, $T=\left\{x \in R \mid x^{2}\right.$ $=0\} \neq\{0\}$, and because $Z W$ is invariant and commutative, there is no loss of generality in assuming that $W$ is a $Z$-module. If $x \in T$, then $1+x \in U$, so $(1+x) w(1-x) \in W$ for each $w \in W$. Thus, $x w-w x-x w x \in W$, and also, $z(x w-w x)-z^{2} x w x \in W$ for any $z \in Z$ since $x$ may be replaced by $z x$. Appropriate $Z$-linear combinations of these two elements yield $\left(z^{2}-z\right)[x, w] \in W$ and $\left(z^{2}-z\right) x w x \in W$. Commute the second of these with $w$ and multiply the resulting equation on the right by $x$ to obtain $\left(z^{2}-z\right) x w x w x=0$. Now $Z \neq$ $\mathrm{GF}(2)$, so a suitable choice of $z \in Z$ and the primeness of $R$ force $x w x w x=0$. But $x r x \in T$ for any $r \in R$. It follows that $(r x w x)^{4}=0$ and $R x w x$ is a nil left ideal of $R$ of index four. By use of Levitzki's Theorem [1, Lemma 1.1, p. 1], we may conclude that $x w x=0$.

Let $r \in R$, and $x, w, y \in W \cap T$. The computation above shows that $\left(z^{2}-z\right)[x r x, w] \in W$, and this element commutes with $y$. The resulting equation, multiplied on the right by wy, gives $\left(z^{2}-z\right) w y x r x w y=0$. As above, we may assume that wyxrwyx $=0$. The fact that $R$ is a prime ring enables us to conclude that $w y x=0$. Now clearly, $W \cap T$ is an invariant set, and $w y(W \cap T)=0$. Hence, the lemma forces either $W \cap T=0$, or $w y=0$, and another application of the lemma in the case $W \cap T \neq 0$ would give a contradiction. Therefore, $W$ contains no nonzero nilpotent element. Since $\left(z^{2}-z\right)[x, w] \in W \cap T(x w x=0)$ for $x \in T$ and $w \in W$, we must have $[x, w]=0$.

Next we show that the nonzero elements of $W$ are regular in $R$. To see this, let $w \in W-(0)$ with $w c=0$ for some $c \in R$. Then $c r w \in T$ for each $r \in R$, and it follows that $c r w^{2}=w c r w=0$. Using the primeness of $R$ again, and that $W \cap T=$ 0 , we may conclude that $c=0$. A similar computation shows that if $c w=0$, then $c=0$. Let $w \in W, r \in R$, and $x \in T$. Then $0=[x r x, w]=x[r, w] x$, so that $x[r, w] \in T$. If $y \in W, 0=[x[r, w], y]=x[[r, w], y]$. This equation and the lemma applied to the invariant set $T$ yield $[[r, w], y]=0$ for $r \in R$ and $w, y \in W$.

Finally, for $w \in W$ and $g \in U,[g, w] g^{-1}=g w g^{-1}-w \in W$, so either $[g, w]=$ 0 , or $[g, w]$ is a regular element of $R$. If $y \in W$, the commutativity of $W$ gives

$$
0=\left[[g, w] g^{-1}, y\right]=[[g, w], y] g^{-1}+[g, w]\left[g^{-1}, y\right]=[g, w]\left[g^{-1}, y\right] .
$$

Therefore, we must have $[g, w]=0$, from which it follows that $W$ centralizes $\bar{U}$. In a prime ring, the centralizer of any nonzero ideal must be central, so $W \subset Z$, completing the proof of the theorem.

Although Theorem 1 is quite special, it can be used to show that suitably restricted invariant subrings are central. We state some corollaries of the theorem, the first of which is an immediate consequence of the observation that the center of an invariant subring is itself invariant. For each corollary we assume that $Z \neq$ GF(2), and that $\bar{U}$ contains a nonzero ideal of $R$. 
COROLlaRY 1. If $W$ is an invariant subring of $R$, then either $Z(W) \subset Z(R)$, or $R$ is a domain.

COROLlaRY 2. If $W$ is an invariant subring of $R$ and contains no nonzero nilpotent element, then $W \subset Z$, or $R$ is a domain.

Proof. Assume that $R$ is not a domain. As in the first part of the proof of Theorem 1, if $x \in T=\left\{r \in R \mid r^{2}=0\right\}$ and $w \in W$, then $x w-w x-x w x \in W$. If char $R \neq 2$, then using $2 x$ instead of $x$ leads to $2 x w x \in W$. Since $W$ contains no nilpotent element other than zero, $x w x=0$. This implies that $[x, w]=0$ as well because $([x, w])^{2}=0$. Should char $R=2$, then $(x w+w x+x w x)^{2}=x w x w+$ $w x w x+x w^{2} x \in W$, and using $x w x \in T$ in the original computation gives $x w x w+$ $w x w x+x w x w x w x \in W$. Adding these two elements yields $x w^{2} x+x w x w x w x \in$ $T \cap W=(0)$. Replacing $x$ by $z x$ for $z \in Z$ and taking a suitable $Z$-linear combination gives $\left(z^{4}+z^{2}\right) x w x w x w x=0$. Use $Z \neq \mathrm{GF}(2)$ to choose a $z \in Z$ with $z^{4} \neq z^{2}$, so that $(x w)^{4}=0$ results. Now replace $x$ with $x r x$ for $x \in R$. As in the proof of Theorem 1, Rxwx is a nil left ideal of $R$ of bounded index, so $x w x=0$ by Levitzki's Theorem [1, Lemma 1.1, p. 1]. Again, we may conclude that $[x, w]=0$. Therefore, $W$ centralizes $T$, and $x w \in T$ for $x \in T$ and $w \in W$. If $y \in W$, then $0=[x w, y]=x[w, y]$, and the lemma applied to $T$ forces $W$ to be commutative. Hence $W \subset Z$ by Theorem 1 .

CoRollary 3. If $W$ is an invariant Artinian subring of $R$, then either $R$ is $a$ domain, $W \subset Z$, or $W=R$.

Proof. Since $W$ is an Artinian ring, its radical $J$ is nilpotent, and clearly invariant, so the lemma gives $J=0$. Assume that $R$ is not a domain. The Wedderburn-Artin structure theory and Corollary 1 show that $W$ must be a simple Artinian ring with $1 \in W$. But now Corollary 2 forces $W \subset Z$, unless $W$ contains nilpotent elements. If $x \in W-\{0\}$ with $x^{2}=0$, then $((1+z x) w(1-z x)-w) x$ $\in W$, for any nonzero $z \in Z$. Thus, $z x w x \in W$, and since $W$ is a simple ring, $x w x \neq 0$ for some $w \in W$. But now $z=z \cdot 1 \in z W=z W x w x W=W(z x w x) W=$ $W$, which shows that $Z=Z(W)$ is a field. Next observe that the existence of nonzero nilpotent elements in $W$ implies that $W$, and so $R$, contains noncentral idempotents. If $E$ is the additive subgroup generated by all idempotents in $R$, it is easy to check that $E$ is a Lie ideal of $R$, and that $L=[E, R]$ is a noncommutative Lie ideal of $R$ contained in the additive subgroup of $R$ generated by $T=\{x \in$ $\left.R \mid x^{2}=0\right\}$ [1, Chapter 1]. Hence, using the initial computation in Theorem 1 again, $[W, L] \subset W$. Now [6, Theorem 14, p. 124] is applicable, and yields either $W \subset Z$, $W$ contains a nonzero ideal of $R$, or that $R$ is four dimensional over its center, $Z$. The last possibility and the simplicity of $W$ give $W=R$. Finally, if $W$ contains an ideal of $R$, then $W$ is an ideal of $R$, so $W=R$ because $1 \in W$.

We return now to our goal of proving that solvable normal subgroups of $R$ must be central. 
THEOREM 2. Let $Z$ contain at least five elements and assume that $\bar{U}$ contains a nonzero ideal of $R$. If $N$ is a solvable normal subgroup of $U$, then either $R$ is a domain or $N \subset Z$.

Proof. Let (1) $=N^{(k+1)} \triangleleft N^{(k)} \triangleleft \cdots \triangleleft N$ be the derived series for $N$. If $k=0$, then $N$ is abelian, so the subring $\bar{N}$ generated by $N$ is commutative and invariant. By Theorem 1 either $N \subset \bar{N} \subset Z$, or $R$ is a domain. If $k>0$, then $N^{(k)}$ is a characteristic subgroup of $N$, so is an abelian normal subgroup of $U$. As we have just seen, $N^{(k)} \subset Z$. Therefore, to contradict $k>0$ and prove the theorem, it suffices to show that if $N \triangleleft U$ with the derived group $N^{\prime} \subset Z$, then $N$ is abelian, unless $R$ is a domain. Henceforth, we assume that $R$ is not a domain, so $T=\left\{x \in R \mid x^{2}=0\right\} \neq\{0\}$.

If $x \in T$ and $g \in N$, then $h=(1+x) g^{-1}(1-x) \in N$, so $g h g^{-1} h^{-1} \in N^{\prime} \subset Z$. A similar element is obtained by replacing $x$ with $z x$ for $z \in Z$. The assumption that $Z$ contains at least four nonzero elements and use of a Vandemonde determinant argument (see [4, Lemma 4.4, p. 556]) yield the following elements in $Z$ :

$$
\begin{aligned}
& f_{1}(x, g)=g x g^{-1}+g^{-1} x g-2 x ; \\
& f_{2}(x, g)=-g x g^{-1} x+g x g^{-2} x g-x g^{-1} x g-g x g^{-1} x-g^{-1} x g x ; \\
& f_{3}(x, g)=x g^{-1} x g x-g x g^{-2} x g x-g x g^{-1} x g^{-1} x g ; \text { and } \\
& f_{4}(x, g)=g x g^{-1} x g^{-1} x g x .
\end{aligned}
$$

Now $f_{4}=0$, since the elements of $Z$ are regular in $R$. In particular $f_{5}(x, g)=$ $x g^{-1} x g^{-1} x g x=0$. Next we compute that $0=x^{2} f_{1}=x f_{1} x=x g x g^{-1} x+x g^{-1} x g x$, multiply this equation on the left by $x g^{-1}$, and use $f_{5}=0$ to obtain $f_{6}(x, g)=$ $x g^{-1} x g x g^{-1} x=x g x g^{-1} x g^{-1} x=0$.

Observe that $f_{3}(x, g) x=-g f_{5}=0$, so the central element $f_{3}=0$. Hence $0=x f_{3}$ $=-x g x g^{-2} x g x-f_{6} g$, and $f_{7}(x, g)=x g x g^{-2} x g x=0$. Using $f_{7}=0$, together with $f_{6}\left(x, g^{-1}\right)=x g x g^{-1} x g x=0$, one obtains $f_{2}(x g x, g)=-g^{-1} x g x g x g x$ is central. The regularity of central elements forces $(x g)^{4}=0$ and replacing $x$ with $x r x$ gives $(r x g x)^{5}=0$. Consequently, $R x g x$ is a nil left ideal of $R$ of index five, so from Levitzki's Theorem [1, Lemma 1.1, p. 1] and the primeness of $R$ we conclude that $x g x=0$.

Since $x g \in T,(1+x g) g(1-x g) g^{-1}=1+[x, g] \in N$, and $[x, g]^{2}=0$. Let $y=$ $[x, g]$ and $u \in U$. Then $v=u(1+y) u^{-1} \in N$, so $v(1-y) v^{-1}(1+y) \in N^{\prime} \subset Z$. As above, similar elements can be obtained by using $z y=[z x, g]$ in place of $y$, for any $z \in Z$. Again, suitable $Z$-linear combinations of these elements show that $z_{1}=y u y u^{-1}-u y u^{-1} y \in Z$ and $z_{2}=\left(u y u^{-1} y\right)^{2} \in Z$. But $y^{2}=0$, so $z_{2}$ is a zero divisor and $y u^{-1} y u y u^{-1} y=0$. This in turn yields $z_{1} y u^{-1} y=y u^{-1} z_{1} y=0$. If $z_{1} \neq 0$, then $y u^{-1} y=0$, so $z_{1} y=0$, and $y=0$ results. On the other hand, if $z_{1}=0$, it follows that any two $U$-conjugates of $y$ commute. Let $W$ be the subring generated by all $U$-conjugates of $y$. Then $W$ is a commutative invariant subring of $R$, so $W \subset Z$ by Theorem 1. But $y \in W$ and $y^{2}=0$, which forces $y=0$. 
We have proved that $[x, g]=0$ for any $g \in N$ and $x \in T$. If $g, h \in N$, then $x g \in T$, so $0=[x g, h]=x[g, h]$. Since $T$ is an invariant subset of $R$, the lemma shows that $N$ is abelian, completing the proof of the theorem.

In [5] it was shown that with our usual hypotheses, if $R$ is a ring with involution, *, then $H=\left\{u \in U \mid u^{*}=u\right\}$ cannot be abelian unless $R$ is a domain or an order in $M_{2}(F)$, for $F$ a field. The question of whether the subgroup generated by $H$ could be solvable remained open. This question can now be answered using Theorem 2 and a remark of Herstein [2, Lemma, p. 268] that the subgroup generated by $H$ is normal in $U$ (for $h \in H$ and $g \in U$, $g h g^{-1}=g h g^{*}\left(\left(g^{-1}\right)^{*} g^{-1}\right)$ ).

THEOREM 3. Let $R$ have an involution, $Z$ have at least five elements, and $\bar{U}$ contain a nonzero ideal of $R$. If the subgroup $N$ generated by the symmetric units in $R$ is solvable, then either $R$ is a domain or an order in $M_{2}(F)$.

Proof. As mentioned above, $N \triangleleft U$ [2], so the solvability of $N$ forces either $N \subset Z$ or $R$ to be a domain, by Theorem 2. In the case $N \subset Z, R$ is either an order in $M_{2}(F)$, or a domain, using [5, Corollary 1, p. 918].

As a final remark, we record the following theorem on invariant subrings which is in the spirit of the main result of [2].

Theorem 4. Let $R$ have an involution, $Z$ have at least five elements, and $R$ contain a noncentral idempotent. If $W$ is a $Z$-submodule of $R$ invariant under conjugation by each symmetric unit, then either $W \subset Z, W \supset[I, R] \neq 0$ for $I$ an ideal of $R$, or $R$ is an order in $M_{2}(F)$. If $W$ is also a subring, the second possibility can be replaced by: $W$ contains a nonzero ideal of $R$.

Proof. Clearly, $W$ is invariant under conjugation by all elements of the subgroup $N$ generated by the symmetric units. Since $N$ is a normal subgroup of $U$ [2], the theorem follows from [6, Theorem 15, p. 124].

\section{REFERENCES}

1. I. N. Herstein, Topics in ring theory, University of Chicago Press, Chicago, III., 1969.

2. A A remark on division rings with involution, Indian J. Pure Appl. Math. 9 (1978), 267-269.

3. L.-K. Hua, On the multiplicative group of a field, Acad. Sinica Sci. Reports 3 (1950), 1-6.

4. C. Lanski, The group of units of a simple ring. I, J. Algebra 15 (1970), 554-569.

5. __ Rings with involution whose symmetric units commute, Canad. J. Math. 28 (1976), 915-928.

6. C. Lanski and S. Montgomery, Lie structure of prime rings of characteristic 2, Pacific J. Math. 42 (1972), 117-136.

Department of Mathematics, University of Southern California, los Angeles, Califormia 90007 